\title{
Is it time for non-alcoholic fatty liver disease screening in patients with type 2 diabetes mellitus?
}

\author{
Giovanni Targher \\ Division of Endocrinology, Diabetes and Metabolism, Department of Medicine, University Hospital of Verona, Verona, Italy \\ Correspondence to: Prof. Giovanni Targher, MD. Section of Endocrinology, Diabetes and Metabolism, University and Azienda Ospedaliera \\ Universitaria Integrata, Piazzale Stefani 1, 37126 Verona, Italy. Email: giovanni.targher@univr.it. \\ Provenance and Peer Review: This article was commissioned by the Editorial Office, Hepatobiliary Surgery and Nutrition. The article did not undergo \\ external peer review. \\ Comment on: Younossi ZM, Golabi P, de Avila L, et al. The global epidemiology of NAFLD and NASH in patients with type 2 diabetes: a systematic \\ review and meta-analysis. J Hepatol 2019;71:793-801.
}

Submitted Sep 30, 2019. Accepted for publication Oct 16, 2019.

doi: 10.21037/hbsn.2019.10.21

View this article at: http://dx.doi.org/10.21037/hbsn.2019.10.21

Non-alcoholic fatty liver disease (NAFLD) has become one of the most important causes of chronic liver disease in Western countries (1). The increasing prevalence of this liver disease parallels the global epidemics of both obesity and type 2 diabetes (T2DM) in many parts of the world (1). $\mathrm{NASH}$ is also projected to become the most common indication for liver transplantation in the near future (1).

A 2016 meta-analysis of 86 epidemiological studies (including a sample size of nearly $8,500,000$ subjects from 22 countries) has estimated that up to a quarter of the world's adult population $[25.2 \%, 95 \%$ confidence intervals (CI): 22.1-28.7\%] has NAFLD (2). This liver disease was common throughout the world, but was particularly frequent in South America (31\%), the Middle East (32\%) and North America (24\%), whereas it showed the lowest frequency in Africa (13\%) (2).

It is well known that NAFLD and T2DM are two pathologic conditions that frequently coexist and act synergistically to increase the risk of adverse clinical outcomes (3). NAFLD may affect up to $70 \%$ of patients with T2DM and up to $30-40 \%$ of those with type 1 diabetes mellitus (3). For example, in 2007 we found that the prevalence of NAFLD (detected by ultrasonography) was extremely common in a cohort of nearly 3,000 Italian patients with T2DM attending a secondary care diabetes service (affecting up to $69.5 \%$ of these patients) (4). Coexisting T2DM not only increases the risk of developing the more severe forms of NAFLD, but also increases the risk of developing hepatocellular carcinoma, liver-related hospitalizations and liver-related deaths (3,5-8). Moreover, the coexistence of NAFLD makes achieving good glycemic control more difficult, exacerbates insulin resistance and promotes atherogenic dyslipidemia, therefore, further increasing the risk of developing major adverse cardiovascular events and chronic kidney disease (CKD), particularly in T2DM patients with advanced NAFLD (3).

Despite the very high proportion and adverse complications of NAFLD in people with T2DM, this liver disease is frequently overlooked in routine diabetes care $(2,4)$. In fact, although T2DM patients have a high proportion of advanced NAFLD, they rarely undergo liver biopsy or imaging techniques for diagnosing and staging NAFLD in clinical practice, since most of these patients with NAFLD have fairly normal serum aminotransferase levels $(3,9)$. Even the 2019 European Society of Cardiology guidelines on diabetes and cardiovascular diseases developed in collaboration with the European Association for the Study of Diabetes do not recommend screening for NAFLD and its liver-related complications in patients with diabetes (10), making the liver a potentially neglected organ and meaning that progression of NAFLD to cirrhosis might be largely undetected in this patient population.

On this background of evidence, the recent metaanalysis written by Younossi et al. (11) is timely and further highlights the importance of increasing awareness of the prognostic value of NAFLD in T2DM patients among primary care physicians, diabetologists/endocrinologists and gastroenterologists/hepatologists. In this updated 
meta-analysis of 80 observational studies from 20 countries (involving a total of nearly 50,000 adult patients with T2DM) the authors aimed at estimating the global prevalence of NAFLD, NASH and advanced fibrosis in people with T2DM (11). Notably, the authors have estimated that the global prevalence of imaging-defined NAFLD in T2DM patients was $55.5 \%$ (95\% CI: $47.3-$ $63.7 \%$ ) with the Europe showing the highest prevalence of NAFLD (68.0\%, 95\% CI: 62.1-73.0\%). In a smaller number of studies estimating the prevalence of biopsyconfirmed NASH and advanced fibrosis the authors found that the global prevalence of these two conditions among patients with NAFLD and T2DM was $37.3 \%$ (95\% CI: $24.7-50 \%$ ) and $17.0 \%$ (95\% CI: $7.2-34.8 \%$ ), respectively (11). Additionally, the authors also reported that over half of patients with NAFLD and T2DM had dyslipidemia, $\sim 60 \%$ had arterial hypertension, $\sim 25 \%$ had cardiovascular disease and $\sim 9 \%$ had peripheral arterial disease (11). These latter results are largely consistent with the findings of previously published studies supporting the additive risk of T2DM and NAFLD, resulting in a higher risk of developing CKD and adverse cardiovascular outcomes $(3,12)$. The risk of developing these extra-hepatic vascular complications parallels the severity of NAFLD histology, such that patients with NASH and varying levels of hepatic fibrosis are at higher risk of incident CKD and adverse cardiovascular outcomes than those with simple steatosis $(3,12)$. It is known that cardiovascular disease is the most predominant cause of mortality in NAFLD patients, independent of other metabolic comorbid conditions. Experimentally, it has been shown that NAFLD, especially in its more advanced forms, releases several proinflammatory, proatherogenic and profibrogenic factors, which may play key roles in the pathophysiology of these extra-hepatic vascular complications $(3,12)$.

Taken together, these findings support the assertion that in patients with T2DM, diagnosis of, and treatment for, NAFLD should be considered a high clinical priority for diabetologists or endocrinologists caring for patients at high risk of progressive NAFLD $(3,9)$. It can, therefore, be argued that there is a case for systematic screening for NAFLD amongst patients at risk of advanced NAFLD, such as those with T2DM. This conclusion is in accordance with the 2016 European (EASL-EASD-EASO) guidelines and the Italian Association for the Study of the Liver guidelines $(13,14)$, but not with the 2016 UK National Institute for Care Excellence (15) or the 2018 American Association for the Study of Liver Diseases (AASLD) guidelines (16). The latter has recommended against a systematic screening for NAFLD in this patient population, because of uncertainties surrounding diagnostic tests and pharmacologic therapies, alongside a lack of knowledge related to the long-term benefits and the cost-effectiveness of screening (16). However, the AASLD practice guidelines have also recommended that there should be a high index of suspicion for NAFLD/NASH in patients with T2DM and that vibration controlled transient elastography in combination with non-invasive markers of fibrosis should be used to identify those patients who are at low or high risk for advanced fibrosis (16).

Personally, I think the possibility of NAFLD should be considered as a part of the routine evaluation in the vast majority of T2DM patients, in the same way we now are searching for cardiovascular disease, CKD and other chronic complications of diabetes. A patientcentred, team-based approach to the management and treatment of patients with T2DM and NAFLD, based on a careful examination of associated cardiometabolic risk factors and monitoring for cardiovascular, renal and liver complications, is warranted. Furthermore, I also think a more widespread use of vibration-controlled transient elastography for identifying T2DM patients at high risk for advanced fibrosis, which is regarded as the strongest determinant of adverse clinical outcomes in NAFLD, might also allow a better choice of the pharmacological treatment for diabetes using some anti-hyperglycemic drugs (if not contraindicated), which have positive effects on NASH and liver fibrosis, such as pioglitazone and liraglutide (and, perhaps, other long-term glucagon-like peptide-1 receptor agonists) (3,9,13-16).

\section{Acknowledgments}

Funding: None.

\section{Footnote}

Conflicts of Interest: The author has completed the ICMJE uniform disclosure form (available at https://hbsn. amegroups.com/article/view/10.21037/hbsn.2019.10.21/coif). The author has no conflicts of interest to declare.

Ethical Statement: The author is accountable for all aspects of the work in ensuring that questions related to the accuracy or integrity of any part of the work are appropriately investigated and resolved. 
Open Access Statement: This is an Open Access article distributed in accordance with the Creative Commons Attribution-NonCommercial-NoDerivs 4.0 International License (CC BY-NC-ND 4.0), which permits the noncommercial replication and distribution of the article with the strict proviso that no changes or edits are made and the original work is properly cited (including links to both the formal publication through the relevant DOI and the license). See: https://creativecommons.org/licenses/by-nc$\mathrm{nd} / 4.0 /$.

\section{References}

1. Byrne CD, Patel J, Scorletti E, et al. Tests for diagnosing and monitoring non-alcoholic fatty liver disease in adults. BMJ 2018;362:k2734.

2. Younossi ZM, Koenig AB, Abdelatif D, et al. Global epidemiology of nonalcoholic fatty liver disease-Metaanalytic assessment of prevalence, incidence, and outcomes. Hepatology 2016;64:73-84.

3. Targher G, Lonardo A, Byrne CD. Nonalcoholic fatty liver disease and chronic vascular complications of diabetes mellitus. Nat Rev Endocrinol 2018;14:99-114.

4. Targher G, Bertolini L, Padovani R, et al. Prevalence of nonalcoholic fatty liver disease and its association with cardiovascular disease among type 2 diabetic patients. Diabetes Care 2007;30:1212-8.

5. Porepa L, Ray JG, Sanchez-Romeu P, et al. Newly diagnosed diabetes mellitus as a risk factor for serious liver disease. CMAJ 2010;182:E526-31.

6. Wild SH, Morling JR, McAllister DA, et al. Type 2 diabetes and risk of hospital admission or death for chronic liver diseases. J Hepatol 2016;64:1358-64.

7. Zoppini G, Fedeli U, Gennaro N, et al. Mortality from chronic liver diseases in diabetes. Am J Gastroenterol

Cite this article as: Targher G. Is it time for non-alcoholic fatty liver disease screening in patients with type 2 diabetes mellitus? HepatoBiliary Surg Nutr 2020;9(2):239-241. doi: 10.21037/hbsn.2019.10.21
2014;109:1020-5.

8. Singal AG, El-Serag HB. Hepatocellular carcinoma from epidemiology to prevention: translating knowledge into practice. Clin Gastroenterol Hepatol 2015;13:2140-51.

9. Bril F, Cusi K. Management of nonalcoholic fatty liver disease in patients with type 2 diabetes: a call to action. Diabetes Care 2017;40:419-30.

10. Cosentino F, Grant PJ, Aboyans V, et al. 2019 ESC Guidelines on diabetes, pre-diabetes, and cardiovascular diseases developed in collaboration with the EASD. Eur Heart J 2020;41:255-323.

11. Younossi ZM, Golabi P, de Avila L, et al. The global epidemiology of NAFLD and NASH in patients with type 2 diabetes: a systematic review and meta-analysis. J Hepatol 2019;71:793-801.

12. Adams LA, Anstee QM, Tilg H, et al. Non-alcoholic fatty liver disease and its relationship with cardiovascular disease and other extrahepatic diseases. Gut 2017;66:1138-53.

13. European Association for the Study of the Liver (EASL); European Association for the Study of Diabetes (EASD); European Association for the Study of Obesity (EASO). EASL-EASD-EASO Clinical Practice Guidelines for the management of non-alcoholic fatty liver disease. J Hepatol 2016;64:1388-402.

14. Italian Association for the Study of the Liver (AISF). AISF position paper on nonalcoholic fatty liver disease (NAFLD): updates and future directions. Dig Liver Dis 2017;49:471-83.

15. Glen J, Floros L, Day C, et al. Non-alcoholic fatty liver disease (NAFLD): summary of NICE guidance. BMJ 2016;354:i4428.

16. Chalasani N, Younossi Z, Lavine JE, et al. The diagnosis and management of nonalcoholic fatty liver disease: Practice guidance from the American Association for the Study of Liver Diseases. Hepatology 2018;67:328-57. 